\title{
THE QUASAR LUMINOSITY FUNCTION
}

\author{
PAUL C. HEWETT \\ Institute of Astronomy \\ Madingley Road, Cambridge CBз OHA, United Kingdom
}

\begin{abstract}
Recent results at high redshifts are briefly reviewed. An analysis of the evolution of the quasar luminosity function based on the recently published Hawkins \& Véron (1995) variability-selected quasar sample is presented. The results support the conclusions of earlier work that suggested the simple two power law luminosity function, of invariant shape as a function of redshift, requires some modification in order to match the data. However, the contention of Hawkins \& Véron that a single power law model provides an adequate fit to the variability-selected quasar sample and that there is no requirement for curvature in the form of the quasar luminosity function is not borne out by the analysis.
\end{abstract}

\section{Introduction}

The number of talks and posters at the 1995 Melbourne IAU Symposium 173, Astrophysical applications of Gravitational Lensing, focusing on the quasar population, whether in the context of strong lensing (multiple images) or weak lensing (quasar-galaxy associations), is significantly smaller than in the previous biennial gravitational lensing meetings. Their place has been taken by reports of new results in areas such as the weak lensing of background sources by galaxy clusters, complete with superb Hubble Space Telescope images, and the extraordinarily impressive results from the various consortia investigating the lensing of stars within our own Galaxy.

The reduced level of reporting that relates to quasar population statistics reflects what could be perceived by many as a lack of recent high-profile activity in the area. Published surveys containing $10^{3}$ quasars now exist and large areas of the redshift-absolute magnitude plane are relatively well populated. Compiling new samples of $\sim 10^{3}$ objects to improve population 
statistics, or undertaking campaigns to improve the quality of observational data (at all wavelengths), do not appear to be receiving high priority. This is perhaps due in part to the realization that observational capabilities are soon to increase by an order of magnitude or more. Planned facilities, notably the $2 \mathrm{dF}$ wide field multi-object spectrograph on the Anglo Australian Telescope, and the SLOAN project, will generate quasar samples with $10^{4}-10^{5}$ objects, dramatically improving the statistics for studies of the evolution, environments and clustering of the quasar population. These projects are likely to have most impact, initially at least, within the apparent magnitude range $16<m_{B}<22$ for objects that are, via their unusual broadband colors for example, relatively easy to detect.

Regions of the redshift-absolute magnitude parameter space not well covered remain so for good reason. In some cases observational campaigns of staggering size are required. For example, essentially all the know highluminosity quasars at significant redshifts have been imaged to search for multiple imaging. However, while quasar surveys at the brightest magnitudes are underway, the very low surface densities and the consequent effort required to compile samples of even tens of quasars at $m<16$, or hundreds at $m<17.5$, means results are very slow to appear. Other portions of the redshift-absolute magnitude plane remain effectively inaccessible. The compilation of a large sample of intermediate luminosity quasars, $M_{B} \sim-24$, at redshifts $z=2-3$, for example, requires the light gathering power and multi-object spectroscopic capability of the $8 \mathrm{~m}$ class telescopes now under construction.

Notwithstanding this rather downbeat assessment of current activity there are important results appearing. Two of these, the recent progress at high-redshifts, and the apparent inconsistency between new results from a large variability-selected sample and the generally accepted picture of quasar evolution, are discussed in this short review.

\section{The High-Redshift Quasar Luminosity Function}

The most distant identifiable collapsed objects, and their use as direct or indirect probes of the early universe, continue to excite interest among astronomers and, notwithstanding the growing number of high-redshift radio galaxies, quasars remain the population identifiable to the highest redshifts. Activity in the 1980s was characterized by a long series of mutually incompatible claims concerning the behavior of the quasar luminosity function at redshifts $z>2.5$ and in particular at redshifts $z>4$. The latter years of the decade saw the instigation of major surveys for quasars at $z \geq 3$. The design of these experiments specifically included, in addition to the capability of simply detecting quasars, the ability to derive the intrinsic space 
density of quasars as a function of absolute magnitude and redshift.

The observational phase of the surveys of Schneider, Schmidt \& Gunn (1994) and of Warren, Hewett \& Osmer (1991) were completed some years ago. An indication of the scale and complexity of calculating a precisely specified selection function for experiments of this type comes from the length of the interval before the groups published their quantitative constraints on the form and normalization of the quasar luminosity function (Warren, Hewett \& Osmer 1994, Schmidt, Schneider \& Gunn 1995). Both groups find evidence for a significant decline in the space density of quasars by $z=4$ although the preferred forms of parameterization are quantitatively different. The agreement between the two surveys is illustrated graphically in Figure 5 of Schmidt et al. (1995). The outcome of the highredshift investigations has demonstrated that complex experiments of this nature can be quantitatively understood and characterized, allowing consistent conclusions to be obtained via entirely independent experimental approaches. Schmidt et al. (1995) summarize the situation: "The excellent agreement between our $z=4$ luminosity function and that of Warren et al. (1994) is remarkable given the very different methods employed". Identical analysis techniques could now be applied using (some) existing as well as new quasar surveys to refine our understanding of the evolution of the quasar luminosity function. At lower redshifts, $z<2$, utilizing the numerically larger samples of quasars available to begin investigating the evolution of the quasar population as a function of quasar spectral energy distribution is a key goal (see Hewett \& Foltz 1994).

\section{The Hawkins' Variability-Selected Quasar Sample}

Hawkins has compiled a unique sample of quasars selected on the basis of their long-term (months to years) photometric variability in the observedframe $B_{J}$ band. Spectroscopically confirmed subsamples of quasars from this work have been available for some time, e.g. Hawkins \& Véron (1993), however, the definition of the published samples employed a flux-limit related to the minimum magnitude achieved by objects over a specified time scale. Although, given the significant level of photometric variability exhibited by the quasars, such a definition has a number of advantages, it has not proved possible to perform a direct comparison of the variability-selected sample with other quasar samples defined using a flux limit at a single epoch. Recently Hawkins \& Véron (1995) published an extensive sample of 315 variability-selected quasars from a single epoch flux-limited object catalogue, making a comparison with other samples possible.

Hawkins \& Véron conclude that the new sample confirms their earlier claims (e.g. Hawkins \& Véron 1993) that there is no evidence for the ex- 


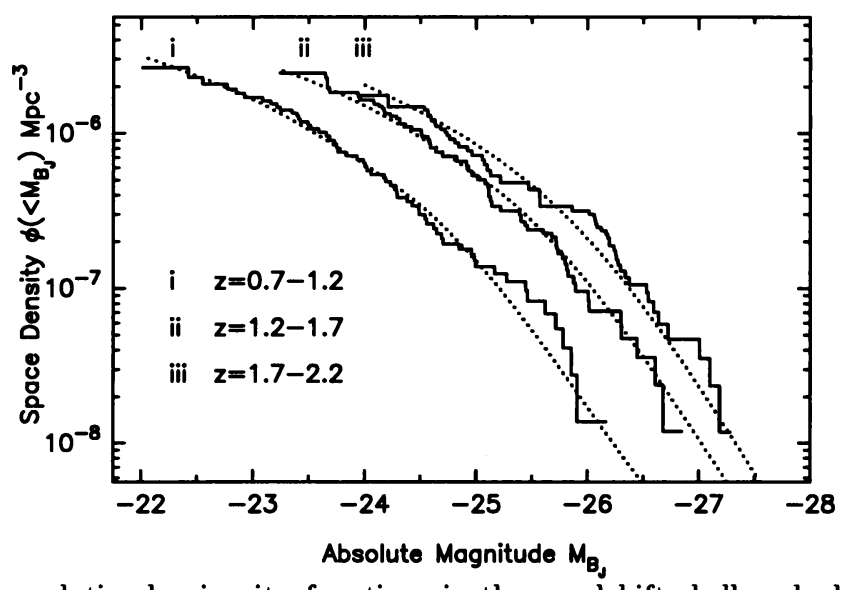

Figure 1. Cumulative luminosity functions in three redshift shells calculated using the Hawkins \& Véron (1995) variability selected sample together with the predictions of a two-power law luminosity function model.

istence of any features in either the quasar number counts or the quasar luminosity function, and they model the quasar luminosity function, calculated for a number of redshift "shells", using a simple power-law form, $\phi=10^{\beta\left(M-M^{*}\right)}$. Their conclusions contrast dramatically with the generally accepted view, based particularly on the data of Boyle et al. (1990), that the quasar luminosity function exhibits a distinct change in shape as a function of absolute magnitude, requiring a model with (for example) two powerlaws of significantly different slope (see Boyle 1993 for a recent review). The potential relevance for gravitational lensing of Hawkins \& Véron's claim is considerable, aside from the substantial implications for the evolution of the quasar population.

The very steep bright-end slope of the differential quasar luminosity function, $N_{q s o}(L) \propto L^{-3.6}$, in the Boyle parameterization and the rapid transition to a much shallower slope, $N_{q s o}(L) \propto L^{-1.5}$, means that strong amplification by gravitational lensing is potentially important at bright magnitudes but becomes essentially insignificant once close to the break luminosity. Hawkins \& Véron's model, which corresponds to $N_{q s o}(L) \propto$ $L^{-2.58}$, would result in amplification by lensing proving to be only moderately important but present for essentially all luminosities. Wu (1994) illustrates the significantly different predictions for the number of quasargalaxy associations that result from the two models.

Figure 1 shows a cumulative representation of the quasar luminosity function calculated using the Hawkins \& Véron sample together with the predictions of a Boyle et al. (1990) two-power law luminosity function un- 


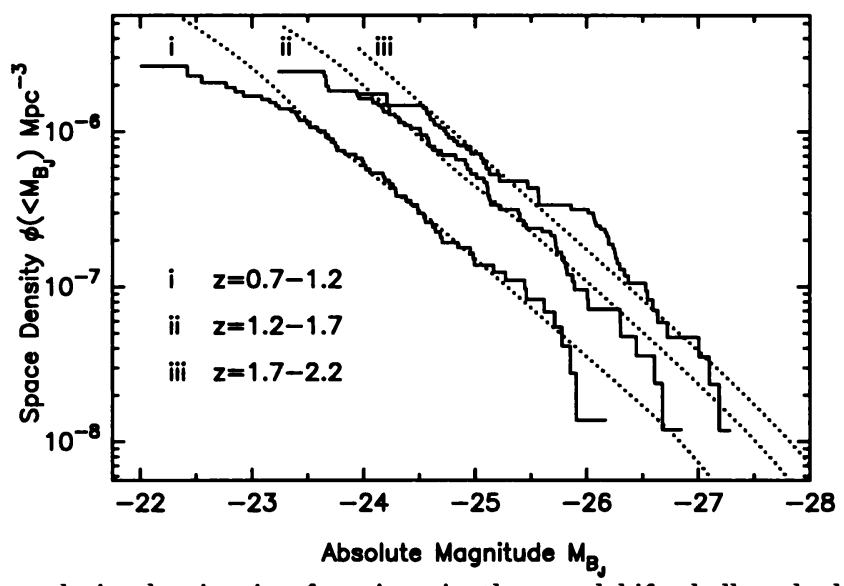

Figure 2. Cumulative luminosity functions in three redshift shells calculated using the Hawkins \& Véron (1995) variability selected sample together with the predictions of a single power law luminosity function model.

dergoing pure luminosity evolution model. A cosmology with $q_{0}=0.5$, $H_{0}=50 \mathrm{~km} \mathrm{~s}^{-1} \mathrm{Mpc}^{-1}$ and $\Lambda=0.0$ was employed. The quasar $k-$ corrections were calculated using the composite quasar spectrum of Cristiani and Vio (1990). None of the conclusions below are sensitive to the adopted (conventional) cosmology or the details of the $k$-corrections.

Luminosity functions were calculated over three redshift shells $z=$ $0.7-1.2$ (57 quasars), $1.2-1.7$ (63 quasars) and $1.7-2.2$ (51 quasars). The solid lines in the figure are derived from calculating accessible volume for each quasar, ordering the quasars by absolute magnitude and plotting the contribution to the space density $\left(=1 / V_{a c c}\right)$. Such cumulative plots have significant advantages over the more prevalent differential representations, particularly when considering samples with small numbers of objects where differential representations necessitate binning over large intervals of absolute magnitude. Note that the contribution of individual objects to the space density is evident as a vertical step at the absolute magnitude of the object. The dashed lines are the predicted distributions of the run of $\Sigma 1 / V_{\text {acc }}$ for a two-power law luminosity function model:

$$
\phi\left(M_{B_{J}}, z\right) d M_{B_{J}} d z=\frac{\phi^{*} d M_{B_{J}} d z}{\left[10^{0.4\left(M_{B_{J}}-M_{B_{J}}(z)\right)(\alpha+1)}+10^{0.4\left(M_{B_{J}}-M_{B_{J}}(z)\right)(\beta+1)}\right]}
$$

with a pure-luminosity evolution of the characteristic magnitude, $M_{B_{J}}(z)$ :

$$
M_{B_{J}}(z)=M_{B_{J}}^{*}-2.5 k_{L} \log (1+z) \quad z<z_{\max }
$$




$$
M_{B_{J}}(z)=M_{B_{J}}\left(z_{\max }\right) \quad z>z_{\max }
$$

with parameters: $\phi^{*}=5.5 \times 10^{-7} \mathrm{mag}^{-1} \mathrm{Mpc}^{-3}, \alpha=-3.9, \beta=-1.5$, $M_{B_{J}}^{*}=-22.5, k_{L}=3.45$ and $z_{\max }=1.7$.

No attempt to achieve a "best-fit" has been made. The two-power law model appears to represent the Hawkins \& Véron data well, particularly the overall curvature. The data in the lowest redshift bin $z=0.7-1.2$ appear to show some evidence for an excess of bright quasars and an improved model fit would be obtained by reducing the amplitude of the slope change in the two power law model. The sense of the deviations from the two-power law model are similar to those found by Hewett, Foltz \& Chaffee (1993) whose analysis of the LBQS sample showed the quasar luminosity function steepening with increasing redshift.

Hawkins \& Véron propose a model for the quasar luminosity function that corresponds to setting $\alpha=\beta=-2.58$. They do not specify what form of evolutionary model they favor and for purposes of illustration the pure luminosity evolution model has been retained. A slightly smaller exponent, $k_{L}=3.20$, is necessary in order to match the observed space densities. The results are shown in Figure 2. The deviations between model and data appear to be larger than for the two-power law model predictions, although a single power law of the slope advocated by Hawkins \& Véron does match substantial portions of the data.

The analysis of the Hawkins \& Véron sample supports the results of earlier work that suggested the simple two power law luminosity function of invariant shape requires some modification. However, the contention that a single power law model provides an adequate fit to the Hawkins variabilityselected quasar sample and that there is no requirement for curvature in the form of the quasar luminosity function is not borne out by the analysis presented here.

\section{References}

Boyle, B.J., 1993, in The Evolution of Galaxies and their Environment ed. H. Thronson and M. Shull (Dordrecht, Kluwer), 433

Boyle, B.J., Fong, R., Shanks, T. \& Peterson, B.A., 1990, MNRAS, 243, 1

Cristiani, S. \& Vio, R., 1990, A\&A, 227, 385

Hawkins, M.R.S.H. \& Véron, P., 1993, MNRAS, 260, 202

Hawkins, M.R.S.H. \& Véron, P., 1995, MNRAS, 275, 1102

Hewett, P.C. \& Foltz, C.B., 1994, PASP, 106,113

Hewett, P.C., Foltz, C.B. \& Chaffee, F.H., 1993, ApJL, 406, L43

Hewett, P.C., Foltz, C.B. \& Chaffee, F.H., 1995, AJ, 109, 1498

Schmidt, M., Schneider, D.P. \& Gunn, J.E., 1995, AJ, 110, 68

Schneider, D.P., Schmidt, M. \& Gunn, J.E., 1994, AJ, 107, 1245

Warren, S.J., Hewett, P.C. \& Osmer, P.S., 1991, ApJS, 76, 23

Warren, S.J., Hewett, P.C. \& Osmer, P.S., 1994, ApJ, 421, 412

Wu, X-P., 1994, A\&A, 286, 748 